PROCEEDINGS OF THE

AMERICAN MATHEMATICAL SOCIETY

Volume 134, Number 7, Pages 1857-1867

S 0002-9939(06)08393-6

Article electronically published on February 17, 2006

\title{
HODGE STRUCTURES ON POSETS
}

\author{
PHIL HANLON
}

(Communicated by John R. Stembridge)

\begin{abstract}
Let $P$ be a poset with unique minimal and maximal elements $\hat{0}$ and $\hat{1}$. For each $r$, let $C_{r}(P)$ be the vector space spanned by $r$-chains from $\hat{0}$ to $\hat{1}$ in $P$. We define the notion of a Hodge structure on $P$ which consists of a local action of $S_{r+1}$ on $C_{r}$, for each $r$, such that the boundary map $\partial_{r}: C_{r} \rightarrow C_{r-1}$ intertwines the actions of $S_{r+1}$ and $S_{r}$ according to a certain condition.

We show that if $P$ has a Hodge structure, then the families of Eulerian idempotents intertwine the boundary map, and so we get a splitting of $H_{r}(P)$ into $r$ Hodge pieces.

We consider the case where $P$ is $\mathcal{B}_{n, k}$, the poset of subsets of $\{1,2, \ldots, n\}$ with cardinality divisible by $k$ ( $k$ is fixed, and $n$ is a multiple of $k$ ). We prove a remarkable formula which relates the characters $\mathcal{B}_{n, k}$ of $S_{n}$ acting on the Hodge pieces of the homologies of the $\mathcal{B}_{n, k}$ to the characters of $S_{n}$ acting on the homologies of the posets of partitions with every block size divisible by $k$.
\end{abstract}

\section{Hodge structures}

Let $P$ be a finite poset with unique minimal and maximal elements $\hat{0}$ and $\hat{1}$. We use the notation $\left(x_{1}, \ldots, x_{r}\right)$ to denote $r$-chains $\hat{0}<x_{1}<x_{2}<\cdots<x_{r}<\hat{1}$. In this case, we will sometimes write $x_{0}$ and $x_{r+1}$ for $\hat{0}$ and $\hat{1}$, respectively. In this paper we will only consider ranked posets, and we will write $r k(x)$ for the rank of $x \in P$.

For each $r$ let $C_{r}$ be a complex vector space with a basis consisting of $r$-chains in $P$. Define $d_{j}: C_{r} \rightarrow C_{r-1}$ by

$$
d_{j}\left(x_{1}, \ldots, x_{r}\right)=\left(x_{1}, \ldots, x_{j-1}, x_{j+1}, \ldots, x_{r}\right) .
$$

Definition 1.1. A local action of $S_{r+1}$ on $C_{r}$ is an action of $S_{r+1}$ on $C_{r}$ such that for each $j \in\{1,2, \ldots, r\}$,

$$
(j, j+1) \cdot\left(x_{0}, x_{1}, \ldots, x_{r}, x_{r+1}\right)=\sum_{x_{j-1}<z<x_{j+1}} c_{z}\left(x_{0}, \ldots, x_{j-1}, z, x_{j+1}, \ldots, x_{r+1}\right)
$$

for suitable constants $c_{z}$.

The idea of local actions on chain spaces of posets originated with Stanley (see [6]).

Received by the editors December 12, 2001 and, in revised form, January 5, 2005.

2000 Mathematics Subject Classification. Primary 05E25.

This work was supported in part by the National Science Foundation under Grant No. DMS0073785.

(C)2006 American Mathematical Society Reverts to public domain 28 years from publication 
Definition 1.2. A Hodge structure on $P$ consists of a collection of local actions of $S_{r+1}$ on $C_{r}$ for all $r=0,1, \ldots, r k(\hat{1})$ which satisfy the following two conditions for all $r$ and all $j \in\{1,2, \ldots, r\}$ :

(a) $(j, j+1) d_{j+1}=d_{j}(j+1, j+2)(j, j+1)$,

(b) $(j, j+1) d_{j}=d_{j+1}(j, j+1)(j+1, j+2)$,

where both sides are considered as linear maps from $C_{r}$ to $C_{r-1}$.

Example 1.3. Fix a positive integer $k$ and let $n$ be a multiple of $k$. Let $P$ be $\mathcal{B}_{n, k}$, the collection of subsets of $\{1, \ldots, n\}$ whose cardinalities are divisible by $k$. For each $A \subseteq B$ in $\mathcal{B}_{n, k}$ define an involution $I_{[A, B]}$ on $(A, B)$ in the following way. If $C \in[A, B]$ write $C=A \cup V$ and $B=C \cup W=A \cup V \cup W$. Then $I_{[A, B]}(C)=A \cup W$. It is straightforward to see that the map $C \rightarrow I_{[A, B]}(C)$ is an involution.

Next, for $\left(A_{1}, \ldots, A_{r}\right)$ and $j \in\{1,2, \ldots, r\}$ define $(j, j+1) \cdot\left(A_{1}, \ldots, A_{r}\right)$ to be $\left(A_{1}^{\prime}, \ldots, A_{r}^{\prime}\right)$, where $A_{\ell}^{\prime}=A_{\ell}$ for $\ell \neq j$ and $A_{j}^{\prime}=I_{\left[A_{j-1}, A_{j+1}\right]}\left(A_{j}\right)$.

Next suppose that $\left(A_{1}, \ldots, A_{r}\right)$ is an $r$-chain in $P$ and that $1 \leq j \leq r-1$. Let $V, W, X$ be such that $A_{j}=A_{j-1} \cup V, A_{j+1}=A_{j} \cup W$ and $A_{j+2}=A_{j+1} \cup X$. Then

$$
\begin{aligned}
(j, j+1)(j+1, j+2)(j, j+1)\left(A_{1}, \ldots, A_{r}\right) & =\left(B_{1}, \ldots, B_{r}\right), \\
(j+1, j+2)(j, j+1)(j+1, j+2)\left(A_{1}, \ldots, A_{r}\right) & =\left(C_{1}, \ldots, C_{r}\right),
\end{aligned}
$$

where $A_{i}=B_{i}=C_{i}$ for $i \notin\{j, j+1, j+2\}$. So we will compare what

$$
(j, j+1)(j+1, j+2)(j, j+1) \text { and }(j+1, j+2)(j, j+1)(j+1, j+2)
$$

do to the subchain $\left(A_{j-1}, A_{j}, A_{j+1}, A_{j+2}\right)$. For simplicity write

$$
\begin{aligned}
A_{j-1} & =Z, \\
A_{j} & =A_{j-1} \cup Y=Z \cup Y, \\
A_{j+1} & =A_{j} \cup X=Z \cup Y \cup X, \\
A_{j+2} & =A_{j+1} \cup W=Z \cup Y \cup X \cup W .
\end{aligned}
$$

Then

$$
\begin{aligned}
& (j, j+1)(j+1, j+2)(j, j+1)\left(A_{j-1}, A_{j}, A_{j+1}, A_{j+2}\right) \\
= & (j, j+1)(j+1, j+2)(Z, Z \cup X, Z \cup X \cup Y, Z \cup X \cup Y \cup W) \\
= & (j, j+1)(Z, Z \cup X, Z \cup X \cup W, Z \cup X \cup W \cup Y) \\
= & (Z, Z \cup W, Z \cup W \cup X, Z \cup W \cup X \cup Y),
\end{aligned}
$$

whereas

$$
\begin{aligned}
& (j+1, j+2)(j, j+1)(j+1, j+2)\left(A_{j-1}, A_{j}, A_{j+1}, A_{j+2}\right) \\
= & (j+1, j+2)(j, j+1)(Z, Z \cup Y, Z \cup Y \cup W, Z \cup Y \cup W \cup X) \\
= & (j+1, j+2)(Z, Z \cup W, Z \cup W \cup Y, Z \cup W \cup Y \cup X) \\
= & (Z, Z \cup W, Z \cup W \cup X, Z \cup W \cup X \cup Y) .
\end{aligned}
$$

This proves that property (a) from Definition 1.2 holds.

To prove property (b), fix $j$ and again let $\left(A_{1}, \ldots, A_{r}\right)$ be an $r$-chain. Then

$$
(j, j+1) d_{j+1}\left(A_{1}, \ldots, A_{r}\right)=\left(B_{1}, \ldots, B_{r-1}\right)
$$


and

$$
d_{j}(j+1, j+2)(j, j+1)\left(A_{1}, \ldots, A_{r}\right)=\left(C_{1}, \ldots, C_{r-1}\right),
$$

where $B_{i}=C_{i}=A_{i}$ for $i<j$ and $B_{i}=C_{i}=A_{i+1}$ for $i>j+1$. So we will consider the effects of $(j, j+1) d_{j+1}$ and $d_{j}(j+1, j+2)(j, j+1)$ on the subchain $\left(A_{j-1}, A_{j}, A_{j+1}, A_{j+2}\right)$. Write $A_{j-1}=Z, A_{j}=Z \cup Y, A_{j+1}=Z \cup Y \cup X$ and $A_{j+2}=Z \cup Y \cup X \cup W$. Then

$$
\begin{aligned}
& (j, j+1) d_{j+1}\left(A_{j-1}, A_{j}, A_{j+1}, A_{j+2}\right) \\
& =(j, j+1)(Z, Z \cup Y, Z \cup Y \cup X \cup W) \\
& =(Z, Z \cup X \cup W, Z \cup X \cup W \cup Y),
\end{aligned}
$$

whereas

$$
\begin{aligned}
& d_{j}(j+1, j+2)(j, j+1)\left(A_{j-1}, A_{j}, A_{j+1}, A_{j+2}\right) \\
& =d_{j}(j+1, j+2)(Z, Z \cup X, Z \cup X \cup Y, Z \cup X \cup Y \cup W) \\
& =d_{j}(Z, Z \cup X, Z \cup X \cup W, Z \cup X \cup W \cup Y) \\
& =(Z, Z \cup X, Z \cup X \cup W, Z \cup X \cup W \cup Y) .
\end{aligned}
$$

This proves condition (b).

Note that condition (b) in the definition of Hodge structure describes how the maps $d_{j}$ relate to the transpositions $(a, a+1)$ when one of $a$ or $a+1$ equals $j$. It is worth recording the relationship for other values of $a$. In these other cases, the maps essentially commute when they act on a chain $\gamma$ because they are acting on disjoint sections of $\gamma$. However, the fact that the map $d_{j}$ reduces the length of $\gamma$ by 1 means that there is a re-indexing of chain elements which must be accounted for in the case that $a>j$. Precisely,

$$
\begin{array}{lll}
d_{j}(a, a+1)=(a, a+1) d_{j} & \text { if } & a<j-1, \\
d_{j}(a, a+1)=(a-1, a) d_{j} & \text { if } & a>j+1 .
\end{array}
$$

The usual poset boundary map $\partial_{r}: C_{r} \rightarrow C_{r-1}$ is given by

$$
\partial_{r}=\sum_{j=1}^{r}(-1)^{j-1} d_{j}
$$

Note that a Hodge structure gives an action of $S_{r+1}$ on the $r$-chains of $P$. The boundary map intertwines the actions of $S_{r+1}$ on $r$-chains and $S_{r}$ on $(r-1)$-chains in some complicated way implied by condition (b). We next try to clarify the relationship between the boundary map $\partial$ and the actions of $S_{r+1}$ and $S_{r}$.

Definition 1.4. For each $r$ and each $i \in\{1,2, \ldots, r\}$ let $\mathcal{S}_{i, r+1-i}$ denote the set of permutations $\sigma \in S_{r+1}$ such that $\sigma(1)<\sigma(2)<\cdots<\sigma(i)$ and $\sigma(i+1)<\sigma(i+2)<$ $\cdots<\sigma(r+1)$. Define $s h_{i, r+1-i}$ to be

$$
s h_{i, r+1-i}=\sum_{\sigma \in \mathcal{S}_{i, r+1-i}} \operatorname{sgn}(\sigma) \sigma .
$$

Lastly, if $P$ is a poset with a Hodge structure, $\alpha$ is an $(i-1)$-chain from $\hat{0}$ to $b$ in $P$ and $\beta$ is an $(r-i)$-chain from $b$ to $\hat{1}$ in $P$, define $\alpha * \beta$ to be

$$
\alpha * \beta=\operatorname{sh}_{i, r+1-i} \cdot(\alpha, \beta),
$$


where $(\alpha, \beta)$ is the $r$-chain in $P$ obtained by concatenating the chain $\alpha$ from $\hat{0}$ to $b$ with the chain $\beta$ from $b$ to $\hat{1}$.

Lemma 1.5. Let $P$ be a poset with a Hodge structure, let $\alpha$ be an $(i-1)$-chain from $\hat{0}$ to $b$ and let $\beta$ be an $(r-i)$-chain from $b$ to $\hat{1}$. Then

$$
\partial(\alpha * \beta)=(\partial \alpha) * \beta+(-1)^{i} \alpha *(\partial \beta) .
$$

Proof. Expanding $\partial(\alpha * \beta)$ we have

$$
\partial(\alpha * \beta)=\sum_{j=1}^{r} \sum_{\sigma \in \mathcal{S}_{i, r+1-i}}(-1)^{j-1} \operatorname{sgn}(\sigma) d_{j} \cdot \sigma(\alpha, \beta) .
$$

The first observation we make is that there is a fundamental cancellation on the right-hand side. Namely, suppose $\sigma$ and $j$ satisfy $\sigma^{-1}(j) \in\{1,2, \ldots, i-1\}$ and $\sigma^{-1}(j+1) \in\{i+1, \ldots, r\}$. Then $\sigma^{\prime}=(j, j+1) \sigma$ is also in $\mathcal{S}_{i, r+1-i}$. Summing the two terms on the right-hand side of (1.2) indexed by $j, \sigma$ and $j, \sigma^{\prime}$ yields

$$
\begin{array}{r}
(-1)^{j-1}\left\{\operatorname{sgn}(\sigma) d_{j} \cdot \sigma(\alpha, \beta)+\operatorname{sgn}\left(\sigma^{\prime}\right) d_{j} \cdot \sigma^{\prime}(\alpha, \beta)\right\} \\
=(-1)^{j-1} \operatorname{sgn}(\sigma) d_{j}(i d-(j, j+1)) \cdot \sigma(\alpha, \beta) \\
=0 .
\end{array}
$$

The latter equality holds because $d_{j}(i d-(j, j+1)) \gamma=0$ for any chain $\gamma$.

In view of (1.3) we can rewrite the right-hand side of (1.2) as

$$
\partial(\alpha * \beta)=\Gamma_{1}+\Gamma_{2},
$$

where

$$
\begin{aligned}
\Gamma_{1} & =\sum_{\substack{\sigma^{-1}(j)<i \\
\sigma^{-1}(j+1)<i}}(-1)^{j-1} \operatorname{sgn}(\sigma) d_{j} \sigma(\alpha, \beta), \\
\Gamma_{2} & =\sum_{\substack{\sigma^{-1}(j)>i \\
\sigma^{-1}(j+1)>i}}(-1)^{j-1} \operatorname{sgn}(\sigma) d_{j} \sigma(\alpha, \beta) .
\end{aligned}
$$

Consider the term in $\Gamma_{1}$ indexed by $j$ and $\sigma$. Let $u=\sigma^{-1}(j)$. By the definition of $\Gamma_{1}, \sigma^{-1}(j+1)<i$, and so (since $\left.\sigma(1)<\sigma(2)<\cdots<\sigma(i)\right), \sigma^{-1}(j+1)=u+1$.

Let $b_{1}, \ldots, b_{\ell}$ be the set of numbers $b$ between $i+1$ and $r+1$ which satisfy $\sigma(b)<\sigma(u)$. Since $\sigma(i+1)<\sigma(i+2)<\cdots<\sigma(r+1)$ we have that $\left\{b_{1}, \ldots, b_{\ell}\right\}=$ $\{i+1, \ldots, i+\ell\}$.

Note that we can write $\sigma$ in the form $\sigma=\tau \omega_{3}$, where $\omega_{3}$ moves the numbers $u+$ $2, \ldots, i$ into their final positions under $\sigma$. In so doing, $\omega_{3}$ will move $\{i+1, \ldots, i+\ell\}$ so that they are positioned immediately to the right of $u+1$ in ascending order. We can then further factor $\sigma$ as $\sigma=\omega_{1} \omega_{2} \omega_{3}$, where $\omega_{2}$ moves $u$ and $u+1$ to the right of $i+1, \ldots, i+\ell$ and where $\omega_{1}$ then puts $i+1, \ldots, i+\ell$ into their final positions under $\sigma$.

To summarize, we can factor $\sigma$ as $\sigma=\omega_{1} \omega_{2} \omega_{3}$, where $\omega_{3}(m)=m$ for $m \leq u+1$, where

$$
\omega_{2}=(j-1, j)(j, j+1)(j-2, j-1)(j-1, j) \cdots(u, u+1)(u+1, u+2)
$$

and where $\omega_{1}(m)=m$ for $m>j-1$. 
By Definition 1.1 we have that $d_{j} \omega_{1}=\omega_{1} d_{j}$. By condition (b) of the definition of the Hodge structure we have

$$
\begin{aligned}
& d_{j} \omega_{2} \\
= & d_{j}(j-1, j)(j, j+1)((j-2, j-1)(j-1, j) \cdots(u, u+1)(u+1, u+2)) \\
= & (j-1, j) d_{j-1}(j-2, j-1)(j-1, j)((j-3, j-2) \cdots(u, u+1)(u+1, u+2)) \\
= & (j-1, j)(j-2, j-1) d_{j-2}(j-3, j-2)(j-2, j-1) \cdots(u, u+1)(u+1, u+2) \\
= & \cdots \\
= & (j-1, j)(j-2, j-1) \cdots(u, u+1) d_{u} .
\end{aligned}
$$

Lastly note that $d_{u} \omega_{3}=\hat{\omega}_{3} d_{u}$, where $\hat{\omega}_{3}$ is obtained from $\omega_{3}$ by removing the fixed point $u$ and reducing numbers greater than $u$ by one.

So $d_{j} \sigma=d_{j} \omega_{1} \omega_{2} \omega_{3}=\omega_{1} \hat{\omega}_{2} \hat{\omega}_{3} d_{u}:=\hat{\sigma} d u$, where $\hat{\omega}_{3}$ is defined above and $\hat{\omega}_{2}=$ $(j-1, j)(j-2, j-1) \cdots(u, u+1)$. Note that $\hat{\sigma} \in \mathcal{S}_{i-1, r-(i-1)}$ and that $\operatorname{sgn}(\hat{\sigma})=$ $\operatorname{sgn}(\sigma)(-1)^{j-u}$, i.e., $(-1)^{j-1} \operatorname{sgn}(\sigma)=(-1)^{u-1} \operatorname{sgn}(\hat{\sigma})$. Thus the summand in $\Gamma_{1}$ indexed by $j, \sigma$ is exactly equal to the summand in $(\partial \alpha) * \beta$ indexed by $u, \hat{\sigma}$.

The identification above is reversible, thus giving a $1-1$ correspondence between summands in $\Gamma_{1}$ and summands in $(\partial \alpha) * \beta$. A similar argument gives a $1-1$ correspondence between summands in $\Gamma_{2}$ and summands in $(-1)^{i} \alpha *(\partial \beta)$. This proves the lemma.

Recall the Eulerian idempotents $e_{n}^{(1)}, \ldots, e_{n}^{(n)}$, which are pairwise orthogonal idempotents in $\mathbb{C} S_{n}$ defined by Gerstenhaber and Schack in [3]. Gerstenhaber and Schack give an abstract definition of the $e_{n}^{(j)}$. Loday [5] gives the following more concrete definition.

For each $n$ and $k$, let $S(n ; k)$ denote the of permutations in $\mathcal{S}_{n}$ with exactly $k-1$ descents. Define elements $\ell_{n}^{(k)}$ and $\psi_{n}^{(k)}$ in $\mathbb{C}\left[S_{n}\right]$ by

$$
\begin{aligned}
\ell_{n}^{(k)} & =(-1)^{k-1} \sum_{\sigma \in S(n ; k)} \operatorname{sgn}(\sigma) \sigma, \\
\psi_{n}^{(k)} & =\sum_{i=0}^{k-1}\left(\begin{array}{c}
n+i \\
i
\end{array}\right) \ell_{n}^{(k-i)} .
\end{aligned}
$$

The following lemma can be used to define the Eulerian idempotents $e_{n}^{(j)}$ :

Lemma 1.6 (Loday, [5]). For every $n$ and $k$ we have

$$
(-1)^{k-1} \lambda_{n}^{(k)}=\sum_{j=1}^{n} k^{j} e_{n}^{(j)}
$$

It is straightforward to see that the equations in Lemma 1.6 determine the $e_{n}^{(j)}$ in terms of the $\lambda_{n}^{(k)}$, since the transition matrix $\left(k^{j}\right)$ is a van der Monde hence invertible.

Let $P$ be a poset with a Hodge structure. Then $C_{r}(P)$ admits an action of $S_{r}$ so $C_{r}(P)$ can be split as a direct sum of subspaces $C_{r}^{(j)}(P)$ where

$$
C_{r}^{(j)}(P)=e_{r}^{(j)} \cdot C_{r}(P) .
$$

Following Gerstenhaber and Schack, Lemma 1.5 implies that

$$
\partial_{r}: C_{r}^{(j)}(P) \rightarrow C_{r-1}^{(j)}(P) .
$$


Thus $H_{r}(P)$ splits as a direct sum of

$$
H_{r}(P)=\bigoplus_{j} H_{r}^{(j)}(P)
$$

where $H_{r}^{(j)}(P)$ is the degree $r$ component of the subcomplex

$$
\cdots \rightarrow C_{r+1}^{(j)}(P) \rightarrow C_{r}^{(j)}(P) \rightarrow C_{r-1}^{(j)} P \rightarrow \cdots
$$

The decomposition above will be referred to as the Hodge decomposition of $H_{*}(P)$. We have proved the following theorem.

Theorem 1.7. Let $P$ be a poset with a Hodge structure. Then the homology of $P$ admits a Hodge decomposition.

In the next section, we look at an example of a poset that admits a Hodge structure and see what we can say about the Hodge decomposition of homology using enumerative and representation theoretic techniques.

\section{2. $k$-DIVISIBLE SUBSETS}

Recall that $\mathcal{B}_{n, k}$ is the collection of subsets of $n$ with cardinality divisible by $k$. In Section 1 we showed that $\mathcal{B}_{n, k}$ ordered by inclusion has a Hodge structure.

In this case, $P=\mathcal{B}_{n, k}$ also admits an action of $S_{n}$ which yields a permutation action of $S_{n}$ on $r$-chains in $P$. From the definition of the Hodge structure given in Example 1.3, it is straightforward to check that

$$
\sigma(j, j+1) \gamma=(j, j+1) \sigma \gamma
$$

for every $\gamma \in C_{r}(P)$, every $\sigma \in S_{n}$ and every $1 \leq j \leq r-1$.

From (2.1), it follows that $S_{n}$ commutes with the projection maps $e_{r+1}^{(j)}$ and so $S_{n}$ acts on the Hodge pieces of $H\left(\mathcal{B}_{n, k}\right)$. Our goal in this section is to learn more about the actions of $S_{n}$ on the Hodge pieces. Note that $\mathcal{B}_{n, k}$ is a ranked poset of rank $\frac{n}{k}$. It is also known that $\mathcal{B}_{n, k}$ is totally semimodular, hence CL-shellable (see [1]). Consequently, the homology of $H_{r}\left(\mathcal{B}_{n, k}\right)$ is 0 except for $r=\frac{n}{k}-1$. Let $\beta_{n, k}^{(j)}$ denote the character of $S_{n}$ on the $j^{\text {th }}$ Hodge pieces of $H_{\frac{n}{k}-1}\left(\mathcal{B}_{n, k}\right)$.

Lemma 2.1. Suppose $k$ divides $n$, let $\beta_{n, k}^{(j)}$ be as above and let $r=n / k$. For $\sigma \in S_{n}$

$$
\beta_{n, k}^{(j)}(\sigma)=\sum_{i=1}^{r}(-1)^{i-1} \sum_{\tau \in S_{r+1-i}}\left[e_{r+1-i}^{(j)}\right]_{\tau}\left|C_{r-i}\left(\mathcal{B}_{n, k}\right)_{(\tau, \sigma)}\right|
$$

where $\left[e_{r+1-i}^{(j)}\right]_{\tau}$ denotes the coefficient of $\tau$ in $e_{r+1-i}^{(j)}$ and where $C_{r-i}\left(\mathcal{B}_{n, k}\right)_{(\tau, \sigma)}$ denotes the set of $(r-i)$-chains in $\mathcal{B}_{n, k}$ which are fixed by $(\tau, \sigma)$. 
Proof. By definition $\beta_{n, k}^{(j)}(\sigma)$ is the trace of $\sigma$ on $H_{\frac{n}{k}-1}^{(j)}\left(\mathcal{B}_{n, k}\right)$. Since the homology of $\mathcal{B}_{n, k}$ vanishes except at the top degree,

$$
\begin{aligned}
\beta_{n, k}^{(j)}(\sigma) & =\sum_{i=1}^{r}(-1)^{i-1} \operatorname{tr}\left(\left.\sigma\right|_{C_{r-i}^{(j)}\left(\mathcal{B}_{n, k}\right)}\right) \\
& =\sum_{i=1}^{r}(-1)^{i-1} \operatorname{tr}\left(\left.\sigma e_{r+1-i}^{(j)}\right|_{C_{r-i}\left(\mathcal{B}_{n, k}\right)}\right) \\
& =\sum_{i=1}^{r}(-1)^{i-1} \sum_{\tau \in S_{r+1-i}}\left[e_{r+1-i}^{(j)}\right]_{\tau} \operatorname{tr}\left(\left.\sigma \tau\right|_{C_{r-i}\left(\mathcal{B}_{n, k}\right)}\right) .
\end{aligned}
$$

The result now follows since $S_{r+1-i} \times S_{n}$ acts on $C_{r-i}\left(\mathcal{B}_{n, k}\right)$ as a group of permutations.

For $\sigma \in S_{p}$, let $j_{i}(\sigma)$ denote the number of $i$-cycles of $\sigma$ acting on $\{1,2, \ldots, p\}$. Define $Z(\sigma)$, the cycle indicator of $\sigma$, by

$$
Z(\sigma)=a_{1}^{j_{1}(\sigma)} a_{2}^{j_{2}(\sigma)} \cdots a_{p}^{j_{p}(\sigma)},
$$

where the $a_{1}, \ldots, a_{p}$ are commuting indeterminates. Lastly, if $\chi$ is a class function on $S_{p}$, define the cycle index of $\chi$ by

$$
Z(\chi)=\frac{1}{p !} \sum_{\sigma \in S_{p}} \chi(\sigma) Z(\sigma) .
$$

Note that $Z(\sigma)=Z(\rho)$ iff $\sigma$ and $\rho$ are conjugate. Also, class functions have constant values on conjugacy classes, so $Z(\chi)$ can be considered to be the generating function of the values of $\chi$.

For each $p$, let $\mathcal{E}_{p}$ denote the trivial representation of $S_{p}$. Following Calderbank, Hanlon and Robinson [2], let $T(0, k)$ denote the sum of the cycle indices of the trivial characters for all symmetric groups $S_{p}$ where $p \equiv 0 \bmod k$. In other words,

$$
T(0, k)=\sum_{n \geq 1} Z\left(\mathcal{E}_{n k}\right)
$$
by

We will use the composition product $A[B]$ for $A, B \in \mathbb{C}\left[\left[a_{1}, a_{2}, a_{3}, \ldots\right]\right]$ defined

$$
A[B]=A\left[a_{i} \leftarrow B\left[a_{j} \leftarrow a_{i j}\right]\right]
$$

where $C[U \leftarrow V]$ denotes substitution of $V$ for every occurrence of $U$.

Lemma 2.2. Fix $k \geq 1$. Let $\tau \in S_{u+1}$. For each $n \geq u$, let $\chi_{n}$ be the class function on $S_{n k}$ whose value on a permutation $\sigma$ is the number of $u$-chains in $\mathcal{B}_{n k, k}$ which are fixed by $(\tau, \sigma)$. Then

$$
\sum_{n} Z\left(\chi_{n}\right)=Z(\tau)[T(0, k)]
$$

Proof. Rewriting the left-hand side of (2.2) we have the equivalent formulation

$$
\sum_{n} \frac{1}{(n k) !} \sum_{\sigma \in S_{n k}}(\# \text { of chains fixed by }(\tau, \sigma)) Z(\sigma)=Z(\tau)[T(0, k)] \text {. }
$$

Let $T_{1}, \ldots, T_{s}$ be the cycles of $\tau$ with $\left[T_{i}\right]=t_{i}$. Suppose $e$ is a chain fixed by $(\tau, \sigma)$, and let $T=\left(u_{1}, \ldots, u_{\ell}\right)$ be a cycle of $\tau$ and let $A_{i}$ be the subset added in $e$ at step 
$u_{i}$. Since $\mathcal{C}$ is fixed by $(\tau, \sigma)$ we must have $\sigma\left(A_{i}\right)=A_{i-1}\left(\right.$ where $\left.A_{0}:=A_{\ell}\right)$. In particular $\left|A_{1}\right|=\left|A_{2}\right|=\cdots=\left|A_{\ell}\right|=m k$ for some $m \geq 1$.

Let $A=A_{1} \cup A_{2} \cup \cdots \cup A_{\ell}$ and let $\sigma_{i}$ be the restriction of $\sigma$ to $A_{i}$. Then $\sigma_{i}$ can be chosen to be any injective function from $A_{i}$ to $A_{i-1}$ and $Z\left(\left.\sigma\right|_{A}\right)=$ $a_{\ell}\left[Z\left(\sigma_{\ell} \sigma_{\ell-1} \cdots \sigma_{1}\right)\right]$. It is straightforward to check that as the $\sigma_{i}$ range over all injective functions from $A_{i}$ to $A_{i-1}$, the product $\sigma_{\ell} \sigma_{\ell-1} \cdots \sigma_{1}$ ranges over all permutations in $S_{m k}$, hitting each one exactly $(m k) !^{\ell-1}$ times. So

$$
\sum_{\sigma_{1}, \ldots, \sigma_{\ell}} Z\left(\left.\sigma\right|_{A}\right)=(m k) !^{\ell-1} \sum_{\sigma \in S_{m k}} a_{\ell}[Z(\sigma)]=(m k) !^{\ell} a_{\ell}\left[Z\left(\mathcal{E}_{m k}\right)\right] .
$$

We now return to the case of $\tau$ which has $s$ cycles $T_{1}, \ldots, T_{s}$. The analysis above allows us to compute the contribution $G\left[m_{1}, \ldots, m_{s}\right]$ made to the left-hand side of (*) by chains $\mathcal{C}$ fixed by a pair $(\tau, \sigma)$, where the subsets at the steps in $T_{i}$ have size $m_{i} k$ (so $n=\left(\sum m_{i} t_{i}\right)$ ). We first choose a subset $S_{i}$ to be divided amongst the steps in $T_{i}$. This can be done in $\left(\begin{array}{c}n k \\ m_{1} t_{1} k, m_{2} t_{2} k, \ldots, m_{s} t_{s} k\end{array}\right)$ ways. Next, for each $i$, we choose to divide the subset into $t_{i}$ equal pieces, and we choose a means to add one at each step of $T_{i}$. This can be done in $\left(\begin{array}{c}m_{i} t_{i} k \\ m_{i} k, m_{i} k, \ldots, m_{i} k\end{array}\right)$ ways. Combining these observations with the analysis above shows that the contribution $G\left[m_{1}, \ldots, m_{s}\right]$ to the left-hand side of $(*)$ is

$$
\begin{aligned}
\frac{1}{(n k) !}\left(\begin{array}{c}
n k \\
m_{1} t_{1} k, \ldots, m_{s} t_{s} k
\end{array}\right) & \prod_{i}\left(\begin{array}{c}
m_{i} t_{i} k \\
m_{i} k, m_{i} k, \ldots, m_{i} k
\end{array}\right)\left(m_{i} k\right) !^{t_{i}} a_{t_{i}}\left[Z\left(\mathcal{E}_{m_{i} k}\right)\right] \\
= & \prod_{i} a_{t_{i}}\left[Z\left(\mathcal{E}_{\left.m_{i} k\right)}\right)\right] .
\end{aligned}
$$

Summing over all $m_{1}, \ldots, m_{s}$ we have that the left-hand side of $(*)$ is given by

$$
\sum_{m_{j} \geq 1} \prod_{i=1}^{s} a_{t_{i}}\left[Z\left(\mathcal{E}_{m_{i} k}\right)\right]=\prod_{i=1}^{s} a_{t_{i}}[T(0, k)]
$$

and this completes the proof of Lemma 2.2 .

We are now ready to state the main result of this section. Recall that $\beta_{n, k}^{(j)}$ denotes the character of $S_{n}$ acting on the $j^{\text {th }}$ Hodge piece of the homology of $\mathcal{B}_{n, k}$.

Theorem 2.3. Fix $k \geq 1$. Then

$$
\sum_{k \mid n}(-1)^{\frac{n}{k}} \sum_{j} \lambda^{j} Z\left(\beta_{n, k}^{(j)}\right)=\prod_{\ell=1}^{\infty}\left(1+a_{\ell}[T(0, k)]\right)^{-\frac{1}{\ell} \sum_{d / \ell} \mu(d) \lambda^{\ell / d}},
$$

where $Z\left(\beta_{0, k}^{(j)}\right)$ is defined to be 1 for $j=0$ and 0 otherwise.

Proof. In 4, Hanlon showed (see formula (6.1) on page 115 combined with Lemma 5.2 on page 113) that

$$
\sum_{j \geq 0} \sum_{p \geq 0} \sum_{\tau \in S_{p}}\left[e_{p}^{(j)}\right]_{\tau} Z(\tau) \lambda^{j}=\prod_{\ell}\left(1+(-1)^{\ell} a_{\ell}\right)^{-\frac{1}{\ell} \sum_{d / \ell} \mu(d) \lambda^{\ell / d}}
$$


Now

$$
\begin{aligned}
& \sum_{k \mid n} \sum_{j} \lambda^{j} Z\left(\beta_{n, k}^{(j)}\right)(-1)^{\frac{n}{k}} \\
= & \sum_{j} \lambda^{j} \sum_{k \mid n} \frac{1}{n !} \sum_{\sigma \in S_{n}} \beta_{n, k}^{(j)}(\sigma) Z(\sigma)(-1)^{\frac{n}{k}} \\
= & \sum_{j} \lambda^{j} \sum_{k \mid n} \frac{(-1)^{\frac{n}{k}}}{n !} \sum_{\sigma \in S_{n}} \sum_{i=0}^{n / k}(-1)^{i-1} \sum_{\tau \in S_{n / k+1-i}}\left[e_{n / k+1-i}^{(j)}\right]_{\tau}\left|\mathcal{C}_{\frac{n}{k}-i}\left(\mathcal{B}_{n, k}\right)_{\tau, \sigma}\right| Z(\sigma) \\
= & \sum_{j} \lambda^{j} \sum_{p=1}^{\infty}(-1)^{p} \sum_{\tau \in S_{p}}\left[e_{p}^{(j)}\right]_{\tau}\left\{\sum_{k \mid n} \frac{1}{n !} \sum_{\sigma \in S_{n}}\left|\mathcal{C}_{\frac{n}{k}-i}\left(\mathcal{B}_{n, k}\right)_{\tau, \sigma}\right| Z(\sigma)\right\} \\
= & \sum_{j} \sum_{p=1}^{\infty} \sum_{\tau \in S_{p}}(-1)^{p}\left[e_{p}^{(j)}\right]_{\tau} \lambda^{j} Z(\tau)[T(0, k)] \\
= & \prod_{\ell}\left(1+a_{\ell}[T(0, k)]\right)^{-\frac{1}{\ell} \sum_{d / \ell} \mu(d) \lambda^{\ell / d}},
\end{aligned}
$$

which completes the proof.

Remarkably, the expression on the right-hand side of Theorem 2.3 appears in another seemingly unrelated context. In particular, the inverse of that expression appears in Theorem 4.6 on page 295 of [2]. We finish this section by describing precisely the relationship implied by this amazing coincidence.

In what follows, $\prod_{n}$ denotes the lattice of set partitions of $\{1, \ldots, n\}$.

Definition 2.4. Let $\alpha \in \prod_{n}$. We say that $\alpha$ is congruent to $i \bmod k$ if every block of $\alpha$ has size congruent to $i \bmod k$. We write this as $\alpha \equiv i(\bmod k)$. Let $\prod_{n}^{(0, k)}$ be the poset of all partitions of $\{1, \ldots, n\}$ congruent to $0(\bmod k)$ together with a minimal element 0 if $n$ is a multiple of $k$. Define $\prod_{n}^{(0, k)}$ to be the empty poset if $n$ is not a multiple of $k$.

It is straightforward to check that $\prod_{n}^{(0, k)}$ is a ranked lattice. The rank function is given by

$$
r(\alpha)= \begin{cases}\left(\frac{n}{k}\right)-\# \text { of blocks }(\alpha)+1 & \text { if } \alpha>0, \\ 0 & \text { if } \alpha=\hat{0} .\end{cases}
$$

Let $P$ be a ranked poset with unique minimal and maximal elements $\hat{0}, \hat{1}$. Let $G$ be a group of automorphisms of $P$. For each $\sigma \in G$ let $P_{\sigma}$ denote the set of elements of $P$ fixed by $\sigma$. Let $\mu_{\sigma}$ denote the Mobius function of $P_{\sigma}$.

Definition 2.5. Let the notation be as above. For each $\sigma \in G$ define the fixed-point characteristic polynomial, $\chi_{\sigma}(\lambda)$, to be

$$
\chi_{\sigma}(\lambda)=\sum_{x \in P_{\sigma}} \mu_{\sigma}(\hat{0}, x) \lambda^{r(\hat{1})-r(x)+1},
$$

where $r$ is the rank function of $P$.

Note that the rank function $r$ used in Definition 2.5) is the rank function of $P$ (in general, $P_{\sigma}$ is not a ranked poset). Note also that $\chi_{\sigma}(\lambda)$, for $\sigma$ the identity of $G$, differs from the usual definition of characteristic polynomial of a poset by a factor of $\lambda$. 
Example 2.6. Let $P=\prod_{6}^{(0,2)}$, let $G=S_{6}$ and let $\sigma=(1,2)(3,4)(5,6)$. Besides the minimal and maximal elements, $P_{\sigma}$ contains three elements at rank 2 $(1234 / 56,1256 / 34,12 / 3456)$ and seven elements at rank $1(12 / 34 / 56,13 / 24 / 56$, $14 / 23 / 56,15 / 26 / 34,16 / 25 / 34,12 / 35 / 46,12 / 36 / 45)$. The value $\mu_{\sigma}(\hat{0}, x)$ of the Mobius function of $P_{\sigma}$ is 2 for $x$ at rank $2,-1$ for $x$ at rank 1 , and 0 for $x=\hat{1}$. Thus $\chi_{\sigma}(\lambda)=\lambda^{4}=7 \lambda^{3}+6 \lambda^{2}$.

We henceforth let $\chi_{\sigma}^{(0, k)}$ denote the fixed point characteristic polynomials for $P=\prod_{n}^{(0, k)}$ and $\sigma \in S_{n}$.

Definition 2.7. Let $\mathcal{R}$ be the set of sequences $\chi=\left(\chi_{0}, \chi_{1}, \chi_{2}, \ldots\right)$, where $\chi_{n}$ is a class function on $S_{n}$ with values in $\mathbb{C}[\lambda]$. There are three natural operations: sum $(+)$, induction product $(*)$ and plethystic product ([]) defined on $\mathcal{R}$ as follows. Let $\chi=\left(\chi_{0}, \chi_{1}, \chi_{2}, \ldots\right)$ and $\psi=\left(\psi_{0}, \psi_{1}, \psi_{2}, \ldots\right)$ be in $\mathcal{R}$. Then

(a) $\chi+\psi=\left(\Gamma_{0}, \Gamma_{1}, \Gamma_{2}, \ldots\right)$, where $\Gamma_{n}=\chi_{n}+\psi_{n}$,

(b) $\chi * \psi=\left(\Gamma_{0}, \Gamma_{1}, \Gamma_{2}, \ldots\right)$, where $\Gamma_{n}=\bigoplus_{d=0}^{n} \operatorname{ind}_{S_{d} \times S_{n-d}}\left(\chi_{d} \otimes \psi_{n-d}\right)$,

(c) $\chi[\psi]=\left(\Gamma_{0}, \Gamma_{1}, \Gamma_{2}, \ldots\right)$, where $\Gamma_{n}=\sum_{d \mid n} \chi_{d}\left[\psi_{n / d}\right]$ and $\chi_{d}\left[\psi_{n / d}\right]$ denotes plethystic product of class functions.

The cycle index of $\underline{\chi} \in \mathcal{R}$ is defined by

$$
Z(\chi)=\sum_{n=0}^{\infty} Z\left(\chi_{n}\right)
$$

It is straightforward to check that

(a) $Z(\chi+\psi)=Z(\chi)+Z(\psi)$,

(b) $Z(\chi * \psi)=Z(\chi) Z(\psi)$,

(d) $Z(\chi[\psi])=Z(\chi)[Z(\psi)]$.

$\mathcal{R}$ together with the operation,$+ *$ is called the induction ring. This is a ring in the usual sense with an identity element $\underline{1}=(1,0,0, \ldots)$, where 1 is the constant function with value $1 . \mathcal{R}$ together with the operations,+[] is called the plethysm ring. It is not a ring in the usual sense, as $A[B+C]$ is not in general equal to $A[B]+A[C]$. However there is an identity for the product [ ] which is the sequence $\left(0, \varepsilon_{1}, 0,0, \ldots\right)$, where $\varepsilon_{1}$ is the trivial representation of $S_{1}$.

Define $\underline{\mathrm{B}}^{(k)}$ and $\underline{\mathrm{C}}^{(k)}$ in $\mathcal{R}$ by

$$
B_{n}^{(k)}(\sigma)=\sum_{j}(-1)^{n / k} \beta_{n, k}^{(j)}(\sigma) \lambda^{j} \text { for } \sigma \in S_{n}, k \mid n,
$$

$\mathcal{C}_{n}^{(k)}(\sigma)=\chi_{\sigma}^{(0, k)}(\lambda)$ for $\sigma \in S_{n}, k \mid n$, and $B_{n}^{(k)}(\sigma)=C_{n}^{(k)}(\sigma)=0$ for $n$ not divisible by $k$.

Theorem 2.8. $\underline{\mathrm{B}}^{(k)}$ and $\underline{\mathrm{C}}^{(k)}$ are inverses in the induction ring.

Proof. By Theorem 2.3 above,

$$
Z\left(\underline{\mathcal{B}}^{(k)}\right)=-\prod_{\ell=1}^{\infty}\left(1+a_{\ell}[T(0, k)]\right)^{-\frac{1}{\ell} \sum_{d / \ell} \mu(d) \lambda^{\ell / d}} .
$$


By Theorem 4.6 on page 295 of [2,

$$
Z\left(\underline{C}^{(k)}\right)=-\prod_{\ell=1}^{\infty}\left(1+a_{\ell}[T(0, k)]\right)^{\frac{1}{\ell} \sum_{d / \ell} \mu(d) \lambda^{\ell / d}} .
$$

So $Z\left(\underline{\mathrm{B}}^{(k)} * \underline{\mathrm{C}}^{(k)}\right)=Z\left(B^{(k)}\right) Z\left(C^{(k)}\right)=1=Z(\underline{1})$, and the result follows.

The proof of Theorem 2.8 fails to give much understanding for why the result is true. It would be very interesting to have a more conceptual or constructive proof of the fact that $\underline{\mathrm{B}}^{(k)} * \underline{\mathrm{C}}^{(k)}=\underline{1}$.

\section{Open PROBlems}

There are a number of other posets which admit a local action on maximal chains. Two examples include the lattice of noncrossing partitions (see 7]) and the posets of shuffles (see [8). In each of these examples, it is not clear how to extend the local action on maximal chains to local action on chains of different lengths. One interesting problem is to define local actions on chains of arbitrary length in these two cases. If that can be done, an interesting question is whether these local actions give a Hodge structure.

A second interesting problem is to give a more constructive proof of Theorem 2.8 .

\section{REFERENCES}

1. A. Bjorner, A. Garsia and R. P. Stanley, "An introduction to Cohen-Macaulay partially ordered sets", (I. Rival, ed., Reidel, Dordrecht, 1982), pp. 583-615. MR0661307 (83i:06001)

2. A. R. Calderbank, P. Hanlon and R. W. Robinson, "Partitions into even and odd block size and some unusual characters of the symmetric groups", Proc. London Math. J. 53 (1986), 288-320. MR0850222 (87m:20042)

3. M. Gerstenhaber and S. D. Schack, "A Hodge-type decomposition for commutative algebra cohomology", J. Pure Appl. Algebra 48 (1987), 229-247. MR0917209 (88k:13011)

4. P. Hanlon, "The action of $S_{n}$ on the components of the Hodge decomposition of Hochschild homology", Michigan Math. J. 37 (1990), 105-124. MR1042517(91g:20013)

5. J.-L. Loday, "Partitions éuleriennes et opérations en homologie cyclique", C. R. Acad. Sci. Paris Sé. I. Math. 307 (1988), 283-286. MR0958781 (89h:18017)

6. R. P. Stanley, "Flag-symmetric and locally rank-symmetric partially ordered sets", Electronic J. Combinatorics 3 R6, 22 pp. MR1392491 (98d:06006)

7. R. P. Stanley, "Parking functions and non-crossing partitions", Electronic J. Combinatorics 4(2) R20, 14 pp. MR1444167 (98m:05011)

8. R. Simion and R. Stanley, "Flag-symmetry of the poset of shuffles and a local action of the symmetric geometry", Discrete Math. 204 (1999), 369-396. MR1691879 (2000f:05090)

Department of Mathematics, University of Michigan, Ann Arbor, Michigan 481091003 